Methods In this project, a questionnaire was developed to predict the risk of long-term sickness absence.

By combining a literature review of the predictive factors for long-term sickness absence, with a review of existing questionnaires that question long-term sickness absence, a new questionnaire was developed. A study has been set up including 10000 participants, to assess the predictive value of the questionnaire and the model to predict the risk of long-term sickness absence.

Results The literature study revealed 16 predictors for long-term sickness absence. The most predictive factor is the patient's expectancy regarding their return to work. As the other predictive value of the other factors was ambiguously, the pilot study will explore the value of the complete model and each separate parameter. The developed questionnaire is not specific for a certain illness, nor for use in a specific country.

Discussion The questionnaire developed in this research aims to support physicians to assess the risk of long-term sickness absence, wherefore an earlier start of return-to work support becomes possible. We aim to guide more employees successfully and sustainably back to work.

\section{RECOGNITION AND SUPPORT OF NURSES IN ACUTE CARE HOSPITALS TOWARD STROKE PATIENTS' RETURN TO WORK}

Yuki Gotoh*, Hitomi Sugisaki, Naomi Yoshikawa, Noriko Hagi. Yokkaichi Nursing and Medical Care University, Yokkaichi, Japan

\subsection{6/oemed-2018-ICOHabstracts. 1574}

Introduction Stroke is a major cause of disability in Japan especially among middle-aged and elderly workers. Nurses take part in patients' care as front-line professionals; thus, they may play an important role in patients' return to work (RTW). However, there is limited research on a clinical nurse's role in RTW. The purpose of this study was to clarify the aspects of recognition and support of nurses in acute care hospitals toward stroke patients' RTW.

Methods Participants were ten nurses with clinical experience of 7 to 20 years. The data were collected using semi-structured interviews conducted from August to September 2014. A Qualitative descriptive approach was employed. The study was approved by the Ethics Committee of the Yokkaichi Nursing Medical University and the hospital.

Result Participants felt they were providing insufficient support for patients' RTW, and that they were busy and providing inadequate care without long-term prospects. Meanwhile, they were aware of the importance of increasing awareness regarding RTW through care that consisted of work and support for patients since hospitalisation. Therefore, they extended support to motivate patients for RTW, and helped expand the activities of daily living and care to increase patients' self-confidence. They also extended the provision of care among other staff through cooperation and coordination. As the care reached and helped the patient and provision of care expanded in the hospital, they realised the rewarding feeling of being a nurse, their recognition as a patient's advocate, and the effect of care. At the same time, they were aware of the importance of prevention and promoting a healthy lifestyle for their patients.
Discussion Nurses were involved in providing care by assessing the occupational background of patients since hospitalisation. Recognition of RTW was deepened by continuous interaction with patients, and the recognition was fostered over a course of time.

\section{SUPPORTING EMPLOYERS DURING RETURN TO WORKOF EMPLOYEES WITH CANCER; DEVELOPMENT OF AN ONLINE INTERVENTION USING THE INTERVENTION MAPPING APPROACH}

${ }^{1} \mathrm{MA}$ Greidanus*, ${ }^{1} \mathrm{AGEM}$ de Boer, ${ }^{2} \mathrm{AE}$ de Rijk, ${ }^{3} \mathrm{CM}$ Tiedtke, ${ }^{3} \mathrm{~B}$ Dierckx de Casterlé, ${ }^{1} \mathrm{MHW}$ Frings-Dresen, ${ }^{1} \mathrm{~S} J$ Tamminga. ${ }^{1}$ Academic Medical Centre, Coronel Institute of Occupational Health, APH, Amsterdam, The Netherlands; ${ }^{2}$ Maastricht University, Department of Social Medicine, CAPHRI, Maastricht, The Netherlands; ${ }^{3}$ Katholieke Universiteit (KU) Leuven, Department of Public Health and Primary Care, Leuven, Belgium

\subsection{6/oemed-2018-ICOHabstracts. 1575}

Introduction Employees with cancer experience difficulties returning to work. Employers play an essential role during return to work (RTW) of employees with cancer, but current RTW interventions pay little attention to the employer. Adequate employer support might be the missing link for successful RTW of employees with cancer. The purpose of this project is therefore to optimise RTW of employees with cancer, by supporting employers.

Methods An online intervention for employers was developed using the Intervention Mapping approach. Firstly, a needs assessment was conducted; the role and support needs of employers during RTW of employees with cancer were assessed (interviews with 30 Dutch employers) and employerrelated perceived barriers and facilitators for work participation of employees with cancer were identified (systematic review) and prioritised (delphi study). Secondly, the objectives of the intervention were specified, practical strategies were chosen, based on interviews with e-health experts, and the online intervention was developed.

Result RTW of employees with cancer requires tailored support from the employer and specific employer/employee communication during different phases:

- disclosure of cancer,

- absence employee during treatment,

- RTW planning,

- actual RTW,

- no return. The review included five and 47 studies representing the employers' and employees' perspectives, respectively.

The identified barriers and facilitators were prioritised by 22 employers and 26 employees with cancer. Subsequently, an online toolbox with 'to-the-point' and tailored support for employers is developed.

Discussion RTW of employees with cancer is a complex trajectory, during which employers play an important role and express a need for support. By involving employers and experts during its development, the online toolbox is expected to fit employers' needs, be feasible in practice and contribute to a successful RTW of employees with cancer. The online toolbox will be evaluated on feasibility in a pilot-RCT with \pm 65 pairs of employer/employee with cancer. 